\title{
Numerical Simulation of Scattering from Rough Surfaces: A Wavelet-Based Approach
}

\author{
Daniel Zahn, Kamal Sarabandi, Senior Member, IEEE, Kazem F. Sabet, Member, IEEE, and \\ James F. Harvey, Senior Member, IEEE
}

\begin{abstract}
In this paper, a preliminary study is carried out to demonstrate the application of wavelets for improving the computation time and reducing computational memory required for evaluating the statistics of the scattered field from rough surfaces using the method of moments (MoM) in conjunction with a Monte Carlo simulation. In specific, Haar and the first order B-spline wavelet basis functions are applied to the MoM formulation of one-dimensional rough surfaces in order to compare the computation time and sparsity for wavelets in the same family but of higher order. Since the scattering coefficient (the second moment of the backscatter field per unit area) is a gentle function of the surface parameters and the radar attributes, it is demonstrated that a relatively high thresholding level can be applied to the impedance matrix, which leads to a sparser impedance matrix and faster computation time. It is also shown that applying a high threshold level the coefficients of the high-order wavelets would increase out of proportion, however, the effect of these current components averages out when computing the scattering coefficients. The resulting sparse impedance matrices are solved efficiently using fast search routines such as the conjugate gradient method. A systematic study is carried out to investigate the effect of different threshold levels on the accuracy versus computing speed criterion. The computed scattering coefficients are compared to previous results computed using a conventional pulse basis function as well as the existing theoretical solutions for rough surfaces. It is shown that wavelet basis functions provide substantial reductions in both memory requirements and computation time.
\end{abstract}

Index Terms-Electromagnetic scattering by rough surfaces, wavelet transforms.

\section{INTRODUCTION}

$\mathbf{T}$ HE problem of electromagnetic scattering from rough surfaces has been the subject of intensive investigation over the past several decades for its application in a number of important remote sensing problems. Radar remote sensing of the oceans, soil moisture, and mine detection using wide-band radars are such examples. For these problems, where the rough surface is either the primary target or the clutter, the understanding of interaction of electromagnetic waves with the rough surface is essential for developing inversion or detection algorithms. An exact analytical solution for random

Manuscript received January 12, 1998; revised October 29, 1999. This work was supported by the U.S. Army Research Office under Contract DAAH 04-960377.

D. Zahn and K. Sarabandi are with the Radiation Laboratory, Department of Electrical Engineering and Computer Science, The University of Michigan, Ann Arbor, MI 48109-2122 USA.

K. F. Sabet is with EMAG Technologies, Inc., Ann Arbor, MI 48105 USA.

J. F. Harvey is with the Army Research Office, Research Triangle Park, NC

27709-2211 USA.

Publisher Item Identifier S 0018-926X(00)01652-5. rough surfaces does not exist. However, approximate analytical solutions exists for rough surfaces with specific types of surface roughness conditions. For surfaces with small root mean square (rms) height and slope, the small perturbation method (SPM) is the most commonly used formulation. Formulations based on SPM exist for perfectly conducting [10], homogeneous dielectric [6], and inhomogeneous dielectric [19] rough surfaces. Another classical solution that is valid for surfaces with large radii of curvature is based on the tangent plane approximation [2]. The region of validity of these classical approaches are rather limited. In recent years, much effort has been devoted to extend the region of validity of these models [1], [12], however, the improved techniques still have the basic limitations of the original models.

An alternative approach for evaluation of the scattered field and its statistics for rough surfaces is Monte Carlo simulation. In this approach, many sample surfaces with the desired roughness statistics are generated and then the scattering solution for each sample surface is obtained using a numerical method. Monte Carlo simulation have primarily been considered for evaluating performance of and characterizing the region of validity of approximate analytical models [1], [12], [22], [23]. In general, the limitations of Monte Carlo simulation of scattering from rough surfaces are the computation time and the required memory as the typical size of the scatterers (sample surfaces) must be chosen to be much larger than the wavelength. Another issue is that the rough surfaces are the targets of infinite extent that must be truncated appropriately before the numerical scattering solution can be obtained. This can be done either using a tapered illumination [1] or padding the sample surfaces with a tapered resistive sheet [7]. It has been shown that with the tapered illumination, larger sample surfaces must be used as a considerable portion of the induced currents on the surface do not contribute significantly to the total scattered field. Application of the tapered resistive sheet is advantageous in that a relatively small portion of the sample surfaces is actually used to suppress the edge currents. This improves the computation time and reduces the required memory.

In order to use Monte Carlo simulation for evaluating the scattering statistics of rough surfaces more routinely, computationally more efficient scattering codes must be developed. In this paper, the application of wavelets as a basis function for the expansion of induced surface currents is considered. Traditional method of moment (MoM) in conjunction with Galerkin's method would require matrix fill computation time of the order of $N^{2}$ and matrix inversion computation time of the order of $N^{3}$ (using Gaussian elimination). It is well 

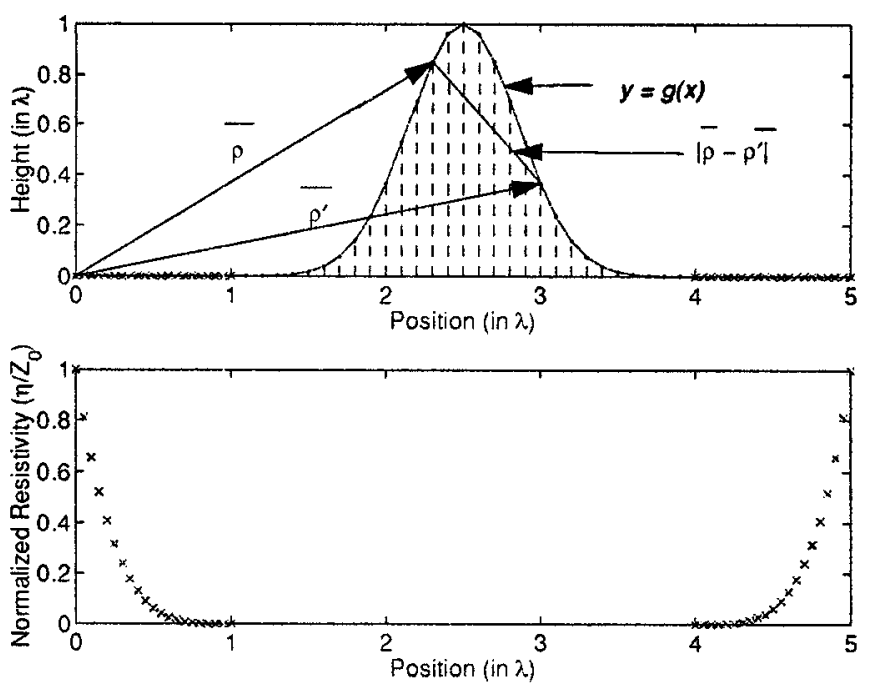

Fig. 1. A typical discretized sample surface with tapered resistive sheets placed at each end ( $1 \lambda$ for each end) and normalized resistivity profile.

known that the solution of linear system of equations can be obtained far more efficiently using search routines such as the conjugate gradient method if the matrix of the coefficients is a sparse matrix. In MoM, the application of conventional pulse or rooftop basis and testing functions would usually produce full impedance matrices. Although the diagonal elements are usually larger than the rest of the elements, the smaller elements cannot be arbitrarily thresholded without drastically altering the resulting scattering pattern. The success of wavelet expansion function in generating sparse matrices have been demonstrated for many circuits and antenna problems [13], [14], [16], [21]. In the Monte Carlo simulation of scattering from rough surfaces, the quantities of interest are the statistical parameters such as the mean and variance of the scattered field and, therefore, it is expected that the overall accuracy be less sensitive to the threshold level.

An investigation is conducted on the use of two different types of wavelets with compact support, Haar and B-spline wavelets with edge wavelets, and the effect of different threshold level with regard to the overall accuracy and the computation time. The method is applied to one-dimensional perfectly conducting random rough surfaces to demonstrate the improvements achieved. In the Monte Carlo analysis presented here, the tapered resistive sheet approach is used to suppress the edge current for plane wave illumination. The numerical results are also compared with the approximate analytical solutions.

\section{INTEGRAL FORMULATION OF SCATTERING PROBLEM}

In order to characterize both the backscattering coefficient and the bistatic scattering coefficient using the MoM and a Monte Carlo simulation, a large number of random surfaces with known statistical parameters $(k s, k \ell)$ must be generated. It is then desired to find the surface current density $\boldsymbol{J}_{e}$ induced by a plane wave from which the scattered field can be computed. The expression for the plane wave propagating along $k_{i}=\sin \theta_{i} \hat{x}-\cos \theta_{i} \hat{y}$ is given by $\boldsymbol{E}^{i}\left(x_{m}, y_{m}\right)=\exp \left(i k_{0}\left(x_{m} \sin \theta_{i}-y_{m} \cos \theta_{i}\right)\right)$.
As discussed in the literature, because of the singular behavior of the current near the edges of the surface when excited by a horizontally polarized incidence wave, tapered resistive sheets must be added at the edges of the sample surface in order to suppress the edge currents. An optimum tapered function for resistivity is given by [7]. Fig. 1 shows a normalized resistivity profile of the tapered resistive sheets and the placement of the resistive sheets on a surface described by a Gaussian hump.

The induced surface current on a resistive sheet is proportional to the tangential electric field or, mathematically, $\hat{n} \times(\hat{n} \times$ $\boldsymbol{E})=-R \boldsymbol{J}$, where $R$ is the surface resistivity (for a perfect conductor $R=0$ ). Another boundary condition for resistive sheets mandates continuity of the tangential electric field across the resistive sheet; that is, $[\hat{n} \times \boldsymbol{E}] \pm=0$.

Therefore, the electric field integral equation for the surface current is given by

$$
\begin{aligned}
\boldsymbol{E}^{i}(\bar{\rho})= & R(\bar{\rho}) J_{e}(\bar{\rho}(\ell))+\frac{k_{0} Z_{0}}{4} \int_{\ell} J_{e}\left(\bar{\rho}\left(\ell^{\prime}\right)\right) \\
& H_{0}^{(1)}\left(k_{0}\left|\bar{\rho}(\ell)-\overline{\rho^{\prime}}\left(\ell^{\prime}\right)\right|\right) d \ell^{\prime}
\end{aligned}
$$

where

$k_{0} \quad$ wave number;

$Z_{0} \quad$ intrinsic impedance of free-space;

$H_{0}^{(1)} \quad$ zeroth-order Hankel function of the first kind;

$\bar{\rho}$ and $\bar{\rho}^{\prime}$ position vectors of observation and source points, respectively.

The sample surface is discretized into sufficiently small cells, as shown in Fig. 1 and the current is expanded in terms of the basis functions $J_{e}(\rho)=\sum a_{n} \phi_{n}(\rho)$. Applying Galerkin's method to (1) we have

$$
\begin{aligned}
& \int_{\ell} \phi_{m}(\bar{\rho}) E^{i}(\bar{\rho}) d \ell \\
&=\sum_{n=1}^{N}\left\{\int_{\ell} a_{n} \phi_{m}(\bar{\rho}) \phi_{n}(\bar{\rho}) R(\bar{\rho}) d \ell+\frac{k_{0} Z_{0}}{4} \int_{\ell} \int_{\ell}\right. \\
&\left.\cdot a_{n} \phi_{m}(\bar{\rho}) \phi_{n}\left(\overline{\rho^{\prime}}\right) H_{0}^{(1)}\left(k_{0}\left|\bar{\rho}-\overline{\rho^{\prime}}\right|\right) d \ell^{\prime} d \ell\right\}
\end{aligned}
$$

where $\phi_{m}(\bar{\rho})$ is the testing function and $\phi_{n}\left(\overline{\rho^{\prime}}\right)$ is the basis function. Equation (2) can be solved using numerical integration, and cast into a matrix equation given by $[Z][I]=[V]$. This matrix equation can easily be solved to find the surface current $\boldsymbol{J}_{e}$.

The restriction on the expansion and testing functions is that they have to be in the domain of the integral equation operation. To expand the induced current in terms of a multiresolution expansion, first the current must be projected onto the $x$-axis or the surface must be arc length parameterized. For natural rough surfaces with moderate rms slope it is more convenient to project the current on the $x$-axis since the domain will be identical for all sample surfaces. Applying a multiresolution expansion to the projected current distribution $(f(x))$ on the $x$-axis we have

$$
\begin{aligned}
f(x) & =\sum_{n} c_{m_{l}, n} \phi_{m_{h}, n}(x) \\
& =\sum_{n} c_{m_{\ell}, n} \phi m_{l}, n(x)+\sum_{m=m_{l}}^{m_{n-1}} \sum_{n} d_{m, n} \psi_{m, n}(x)
\end{aligned}
$$



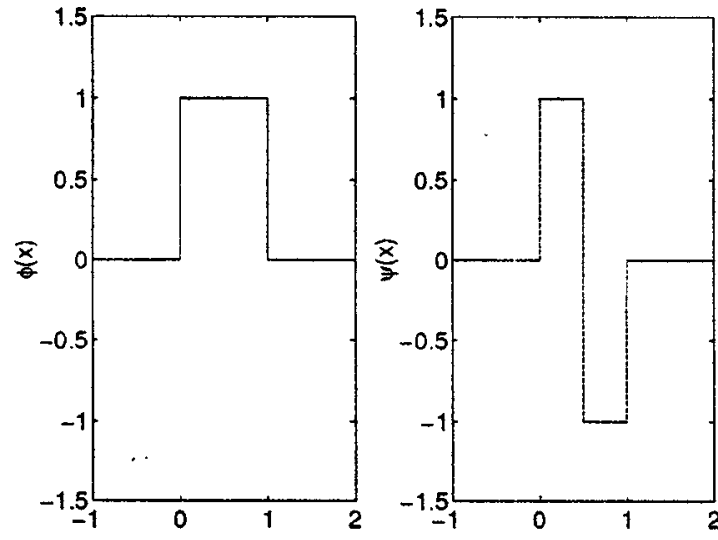

(a)
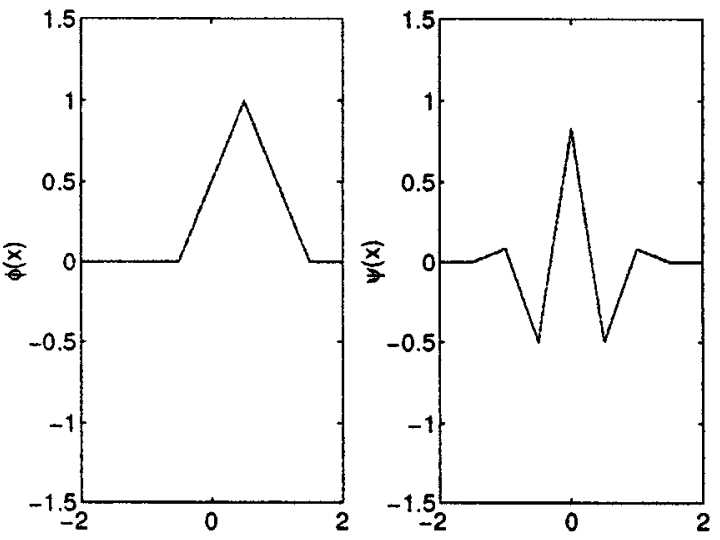

(b)

Fig. 2. (a) Pulse basis function and the corresponding Haar wavelet and (b) linear basis function and its corresponding wavelet.

which consists of the scaling functions at the lowest resolution, plus wavelets at the lowest resolution and subsequent higher resolutions. This expansion is equivalent to one consisting of only scaling functions at the highest resolution $\left(\phi_{m_{h}, n}(x)\right)$. Note that the scaling functions resolution level $m_{l}$ and the wavelets at resolution levels $m_{l} \leq m \leq m_{h}-1$ are orthogonal to each other [15].

By definition, wavelets have a zero average. Higher order wavelets also have vanishing higher moments, that is

$$
\int x^{n} \psi(x) d x=0 \quad n \in\{0, \cdots, N\}
$$

where $N$ depends on the order of the B-spline wavelet. Fig. 2(a) and (b) shows the Haar and linear B-spline scaling functions and wavelets. The wavelets' vanishing moments give rise to their cancellation effect, which makes wavelet basis functions poor radiators. In the moment matrix, this effect leads to very small matrix elements for cells that are relatively far from one another, where the Green's function kernel is fairly regular. For self cells and adjacent cells, the integration of the rapidly varying kernel produces element values that are significant. Thus, due to the cancellation effect, a wavelet-dominated moment matrix tends to be highly sparse. For more insight into wavelet basis functions and matrix sparseness, the reader is referred to [17].

Another important feature of wavelets that arises from their vanishing moment property is their ability to characterize discontinuities and rapid variations. In other words, in a multiresolution expansion, the wavelet coefficients are significant in magnitude only at discontinuities and regions with rapid variations. When the expansion involves multiple resolution levels, the wavelets will capture the information of details at different scales, while the scaling functions will carry average information on the lowest resolution [3]. When the physical problem at hand involves many different scales including large smooth regions and localized sharp variations, a multiresolution expansion will prove the most efficient approximation because the basis functions will carry the maximum localized information without considerable interaction with one another.

Using the fast wavelet transform (FWT), one can compute an equivalent impedance matrix for the multiresolution expansion
(5) from a knowledge of the impedance matrix at the highest resolution [15]. This reduces computation time drastically and avoids performing the numerical integration for all the wavelets at various resolution levels. Once the wavelet-dominated matrix is generated, a threshold level, usually of the order of 0.01 to $1 \%$, is imposed on the matrix and only the significant elements of the matrix will be preserved. Having a sparse impedance matrix, a fast sparse-based solver such as conjugate gradient method can be used to find the solution of the linear system. With the combination of sparse storage schemes and sparse linear solvers, the computation time for linear system inversion is reduced drastically.

\section{Monte Carlo Simulations of Random Rough SURFACES}

Encouraged by previous results, as in [21] where a simple structure has been studied (a double slot aperture) and the current distributions calculated from sparse matrices are compared to the actual (magnetic) current distribution and found to agree quite well, we now apply the multiresolution expansion method to random rough surfaces of known statistical parameters. Near normal incidence, sample surfaces for numerical analysis must be at least 40 correlation lengths $(\ell)$ long in order to accurately characterize the bistatic pattern. This requirement becomes more stringent for near grazing incidence.

Since the Monte Carlo simulation of the scattering problem requires the numerical solution of the problem many times, the computation speed achieved by thresholding the moment matrix of a multiresolution expansion in conjunction with a search routine linear system solver becomes very significant with regard to the overall computation time necessary for evaluating the statistics of the scattered field.

\section{A. Random Surface Generation}

Monte Carlo simulations require a large number of sample surfaces of a random process with prescribed surface height statistics. To generate the sample surfaces, the procedure in [2], [10], is used. First, a long string of numbers is generated using 


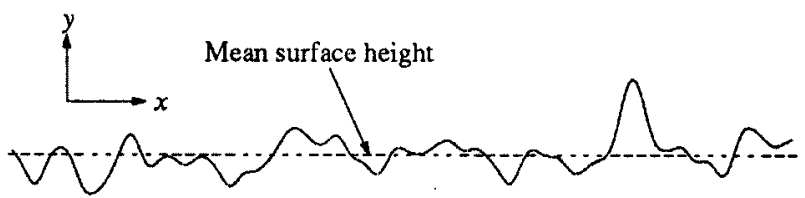

(a)

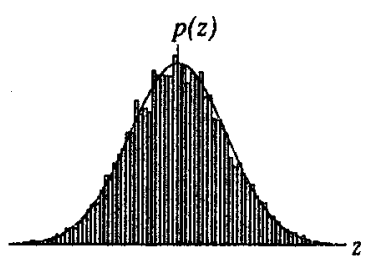

(b)

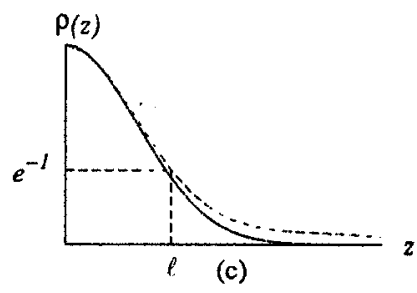

Fig. 3. (a) Generated random surface with mean surface height shown. (b) Theoretical and Monte Carlo probability density function of the surface height distribution ks. (c) Theoretical and Monte Carlo correlation function.

a random number generator having the same pdf as the height distribution of the surface [for example, a zero-mean Gaussian probability density function (pdf)]. Next, a subset of the numbers of the string are correlated with a weight vector related to the Fourier transform of the desired autocorrelation function [1]. For the simulations presented in this paper, surfaces with Gaussian correlation function and Gaussian height distribution are considered. Hence, the surface statistics are uniquely specified by the surface height standard deviation (rms height) $s$, and by the surface correlation length $\ell$. Fig. 3 shows a sample surface of a Gaussian process with $k s=0.3, k \ell=3.0$ and the corresponding histogram of height and calculated correlation function generated from 60 independent sample surfaces. The calculated correlation function gives $k \ell=3.17$ and the calculated standard deviation of height distribution gives $k s=0.3020$. These agree closely with the desired surface roughness parameters.

\section{B. Validation and Results}

Numerical simulation of rough surface scattering is performed for three different surfaces denoted by $S_{1}, S_{2}$, and $S_{3}$. The roughness parameters $(k s, k \ell)$ for each of these surfaces are, respectively, $k s_{1}=0.3, k \ell_{1}=3.0 ; k s_{2}=0.5$, $k \ell_{2}=6.13$; and $k s_{3}=2.0, k \ell_{3}=2.5$. The first two surfaces fall within the region of validity of small perturbation and physical optics (PO) models, respectively, and, hence, the two numerical results can be compared with the analytical models. Comparisons are also made on the threshold level imposed on the moment matrix for both Haar and B-spline wavelet basis functions and on the matrix solving times using a fast conjugate gradient solver for sparse matrices. Another test that was run was the effect of the MoM and wavelet technique on backscattering enhancement. Finally, the effect of the number of resolution levels on the scattering pattern is investigated.

SPM is known to be valid when $k s \leq 0.3, k \ell \leq 3.0$, and $m \leq 0.3$, where $m$ is the rms slope and is given by $m=\sqrt{2} s / \ell$ for a surface with a Gaussian correlation function. The analytical bistatic pattern for the SPM is derived in [24] for a three-dimensional (3-D) surface with a Gaussian correlation function.

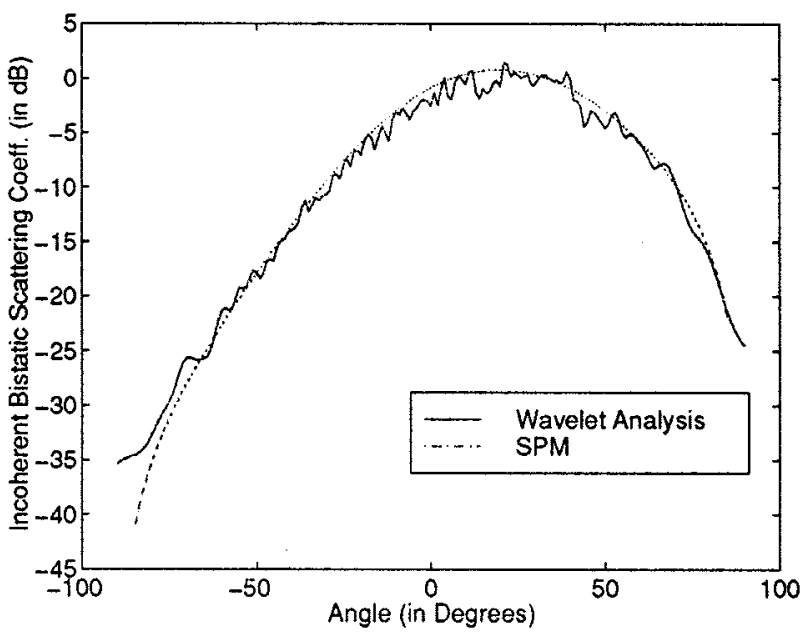

Fig. 4. Comparison of SPM with Monte Carlo simulation. Generated surface parameters are: $k s=0.3, k \ell=3.0, N=40$, length $=32 \lambda$, resistive tapered ends length $=1 \lambda, \Delta x=0.1 \lambda$, and $\theta_{i}=30.0^{\circ}$.

For a two-dimensional (2-D) surface, the bistatic scattering coefficient $\left(\sigma_{2 d}^{\circ}\right)$ is related to the 3-D bistatic scattering coefficient $\left(\sigma_{3 d}^{\circ}\right)$ via $\sigma_{2 d}^{\circ}=(\pi / k) \sigma_{3 d}^{\circ}$. Then, $\sigma_{2 d}^{\circ}$ is given by

$$
\sigma_{2 d}^{\circ}\left(\theta_{i}, \theta_{s}\right)=4 k^{3} \cos ^{2}\left(\theta_{s}\right) \cos ^{2}\left(\theta_{i}\right) f_{h h} W\left(\left|k_{\perp}-k_{\perp i}\right|\right)
$$

where $W(k)$ is the power spectral density. For a Gaussian surface correlation, $W\left(\left|k_{\perp}-k_{\perp i}\right|\right)$ is given by

$$
\begin{aligned}
& W\left(\left|k_{\perp}-k_{\perp i}\right|\right) \\
& \quad=\sqrt{\pi} \ell s^{2} \exp \left(-\left(\frac{(k \ell)^{2}}{4}\left[\sin \left(\theta_{s}\right)-\sin \left(\theta_{i}\right)\right]^{2}\right)\right) .
\end{aligned}
$$

Furthermore, for a perfect electrical conductor (PEC), $f_{h h}=$ $\cos ^{2}\left(\phi_{s}-\phi_{i}\right)$ equals one for a 2-D problem.

The incoherent bistatic scattering coefficient for $\theta_{1}=30^{\circ}$ using the Monte Carlo simulation with 40 independent samples and threshold applied is compared to the analytical bistatic SPM from (5) in Fig. 4 and a good agreement is observed.

The PO method using the tangent-plane technique for approximating the fields on a surface $S$ is investigated next. Under the tangent-plane technique, the fields present at any point $P$ on $S$ are approximated by the fields that would be present on a plane tangent to $P$. This is a valid approximation if every point on $S$ has a large radius of curvature. The $3-\mathrm{D}$ bistatic scattering coefficient is derived in [4] for the PO approximation. Using this formulation $\sigma_{2 d}^{\circ}$ is calculated and is given by

$$
\begin{aligned}
& \sigma_{2 d}^{\circ}\left(\theta_{i}, \theta_{s}\right) \\
& \quad=k\left(\frac{1+\cos \left(\theta_{i}\right) \cos \left(\theta_{s}\right)-\sin \left(\theta_{i}\right) \sin \left(\theta_{s}\right)}{\cos \left(\theta_{i}\right)+\cos \left(\theta_{s}\right)}\right)^{2} J
\end{aligned}
$$

where

$$
\begin{aligned}
J= & 2 \int_{0}^{\infty} d x \cos \left[k x\left(\sin \left(\theta_{i}\right)-\sin \left(\theta_{s}\right)\right)\right] \\
& \cdot\left(\exp \left(-\chi^{2}\left[1-\exp \left(-\frac{x^{2}}{\ell^{2}}\right)\right]\right)-e^{-\chi^{2}}\right)
\end{aligned}
$$

where $\chi=k s\left[\cos \left(\theta_{i}\right)+\cos \left(\theta_{s}\right)\right]$. For surface $S_{2}$, which falls into the $\mathrm{PO}$ region of validity, a comparison is made in Fig. 5 between the PO model in (7) and results obtained using the wavelet 


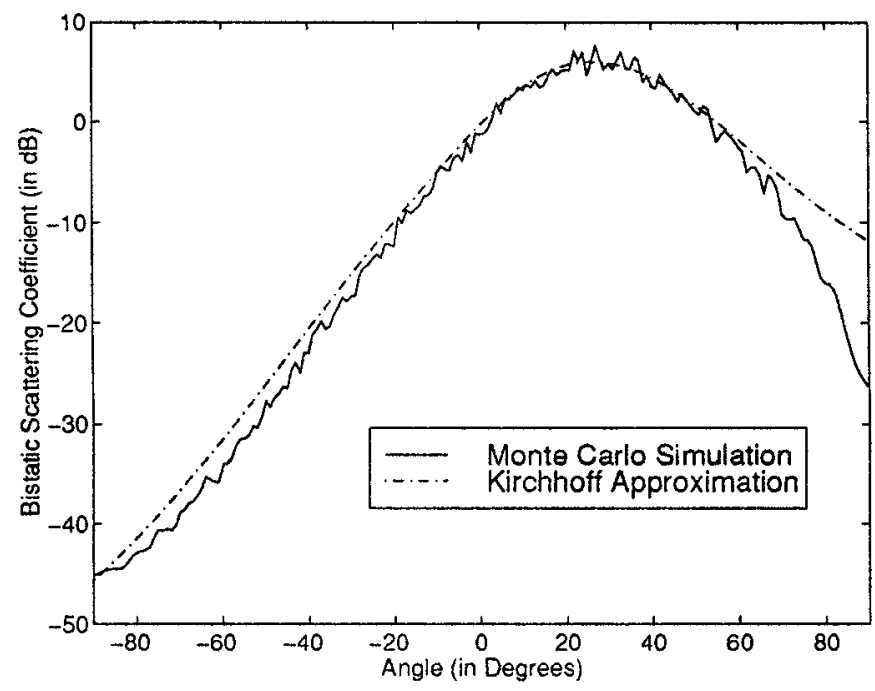

Fig. 5. Comparison of the PO model to a Monte Carlo simulation. For the simulation the random surfaces were characterized by $k s=0.5, k \ell=6.13$, $N=50$, length $=100 \lambda$, resistive tapered ends length $=1 \lambda, \Delta x=0.2 \lambda$, and $\theta_{i}=30.0^{\circ}$.

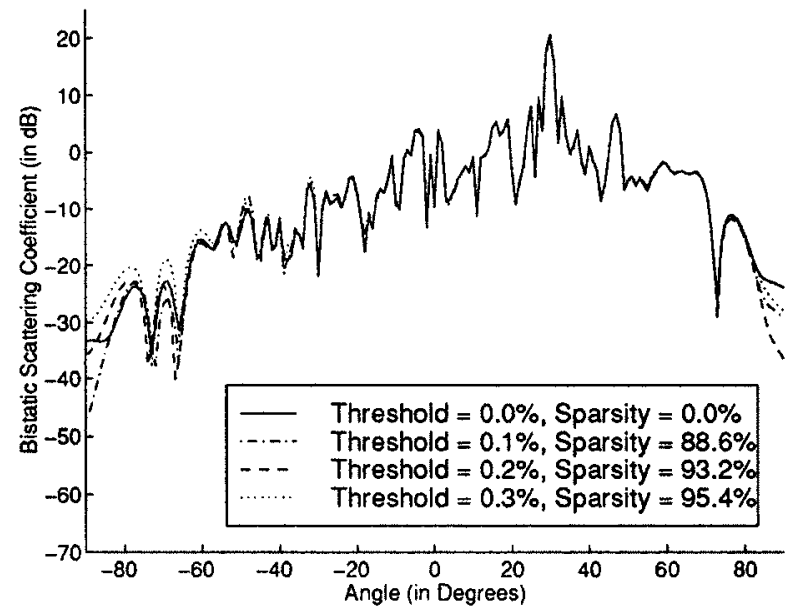

Fig. 6. Bistatic scattering from a single random surface with $k s=0.3, k \ell=$ 3.0, $N=1$, length $=32 \lambda$, resistive tapered ends length $=1 \lambda, \Delta x=0.1 \lambda$, and $\theta_{i}=30.0^{\circ}$. The various threshold levels imposed on the moment matrix and its corresponding sparsity level are given in the figure.

based moment method. The bistatic scattering results from $S_{2}$ agree well with the theoretical PO solution.

A comparison of different threshold levels imposed on the moment matrix for surface $S_{1}$ using B-spline basis functions is shown in Fig. 6. The parameters for the simulation on $S_{1}$ are $\theta_{i}=30.0^{\circ}$, length $=32 \lambda$, resistive tapered ends length $=1 \lambda$ and $\Delta x=0.1 \lambda$. For this simulation, only a single surface $(N=1)$ is used for comparison. As is shown in Fig. 6, the scattering pattern varies very slightly and only at angles near grazing observation. This figure indicates that a sparsity of more than $90 \%$ can be achieved without substantial compromise in the accuracy of the bistatic pattern using B-spline wavelets.

The bistatic scattering coefficient obtained using the Haar and B-spline wavelet of surface $S_{3}$ are shown in Fig. 7. For this simulation, $k s=2.0, k \ell=2.5$, number of independent sur-

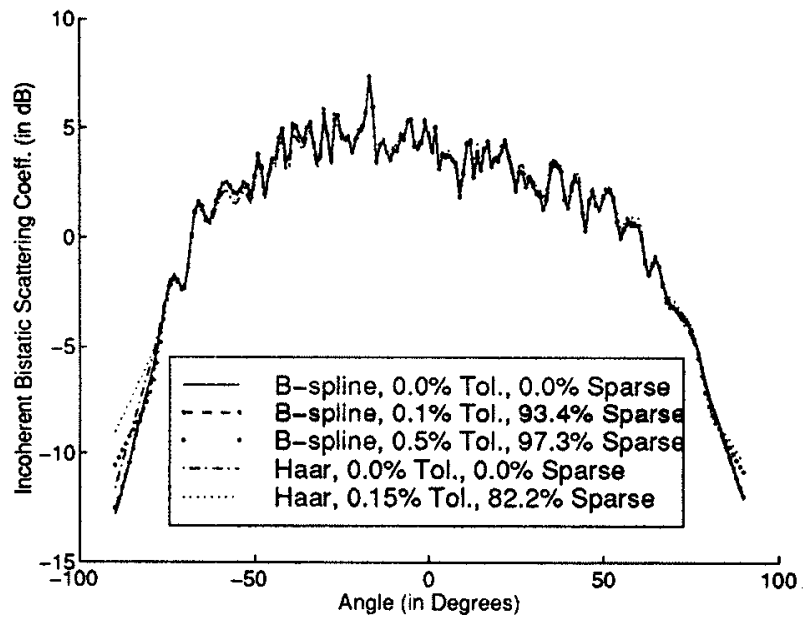

Fig. 7. Comparison of threshold levels on scattering pattern for a random surface with $k s=2.0, k \ell=2.5, N=40$, length $=32 \lambda$, resistive tapered ends length $=1 \lambda, \Delta x=0.04 \lambda$, and $\theta_{i}=30.0^{\circ}$, and five levels of resolution.

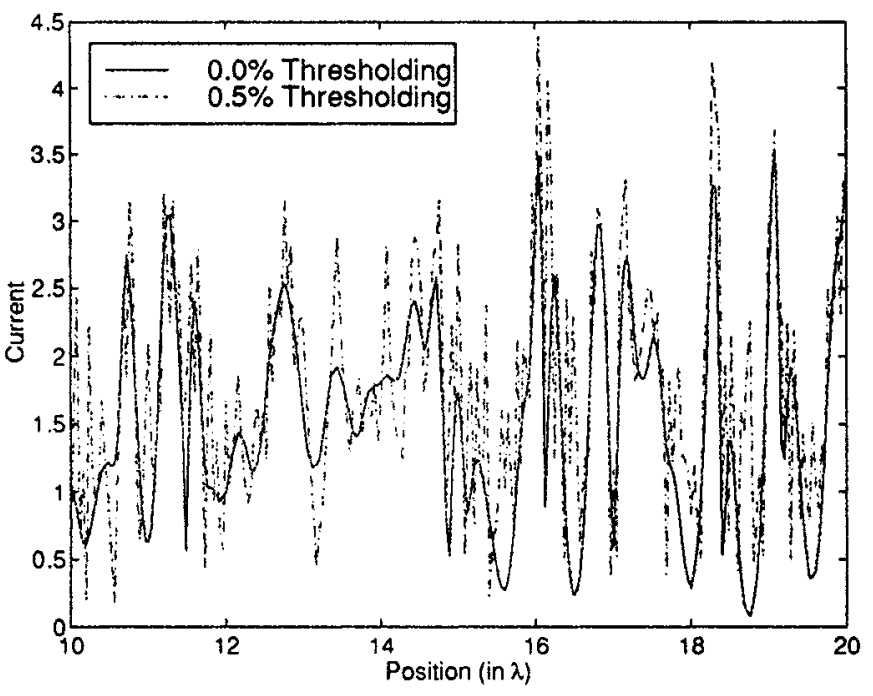

Fig. 8. Comparison of exact current distributions with the current distribution from an impedance matrix that is about $97 \%$ sparse (using B-spline wavelets and a threshold of $0.5 \%$ ). A portion of a random surface with $k s=2.0, k \ell=$ 2.5 , length $=32 \lambda$, resistive tapered ends length $=1 \lambda, \Delta x=0.04 \lambda$, and $\theta_{i}=30.0^{\circ}$, and five levels of resolution is plotted.

faces $N=40$, sample length $=32 \lambda$, resistive tapered ends length $=1 \lambda, \Delta x=0.04 \lambda$, and $\theta_{i}=30.0^{\circ}$. Since this surface does not fall into the region of validity for any analytical models, no comparison may be made. This figure shows again that by thresholding the moment matrix, a high sparsity can be achieved without compromising the accuracy. It is also shown that a higher sparsity is achieved using the linear B-spline wavelet. An average sparsity of $97.3 \%$ is achieved by the linear B-spline wavelet, whereas using Haar wavelets, only $82.2 \%$ average sparsity is obtained for a threshold level of $0.15 \%$. Fig. 8 shows a comparison of the exact current distribution and one where the impedance matrix has a sparsity of about $97 \%$. A portion of a surface with characteristics given above $(k s=2.0$, $k \ell=2.5$, etc.) was used and the sparse matrix has a threshold of $0.5 \%$. Even though this sample surface (which is indicative 
TABLE I

Number OF MULTiPLiCATIONS NEEDED TO

SOLVE THE MATRIX AND SPARSITY VERSUS IMPOSED THRESHOLD LEVEL FOR A RANDOM SURFACE WITH $k s=2.0, k \ell=2.5, N=40$, LENGTH $=32 \lambda$,

RESISTIVE TAPERED ENDS LENGTH $=1 \lambda, \Delta x=0.04 \lambda, \theta_{i}=30.0^{\circ}$, AND FiVE LEVELS OF RESOLUTION

\begin{tabular}{c|c|c|c}
\hline & Threshold & Sparsity & Number of Mults \\
\hline Bspline & $0.0 \%$ & $0.0 \%$ & $5.3 \times 10^{8}$ \\
Bspline & $0.05 \%$ & $90.7 \%$ & $8.4 \times 10^{7}$ \\
Bspline & $0.1 \%$ & $93.4 \%$ & $6.4 \times 10^{7}$ \\
Bspline & $0.5 \%$ & $97.3 \%$ & $2.6 \times 10^{7}$ \\
Haar & $0.0 \%$ & $0.0 \%$ & $7.0 \times 10^{8}$ \\
Haar & $0.05 \%$ & $66.1 \%$ & $9.5 \times 10^{8}$ \\
Haar & $0.1 \%$ & $76.9 \%$ & $7.0 \times 10^{8}$ \\
Haar & $0.15 \%$ & $82.2 \%$ & $5.6 \times 10^{8}$ \\
\hline
\end{tabular}

of the current distribution for every sample surface) has a current distribution that varies significantly from the exact solution, because the far-field scattering pattern is an averaging process the bistatic scattering pattern does not vary significantly from Fig. 7. As stated in Section II, the wavelets capture the information of details with respect to the problem scale. Since the surface is discretized by a maximum of $\lambda / 5$, and usually no more than $\lambda / 10$, the wavelets capture most of the current information in lower resolution wavelets. Thus, most of the higher frequency wavelets may be discarded without significantly altering the bistatic scattering pattern, and this leads to higher sparsity for matrices that use higher resolution wavelets.

The threshold level imposed on the moment matrix and the number of multiplications needed to solve the matrix using a fast conjugate gradient solver routine is studied next. As stated previously, by imposing a threshold level the scattering pattern remains relatively unchanged, yet the moment matrix could be made quite sparse. It was also found that linear B-spline wavelets would produce a sparser matrix for a given threshold level before the scattering pattern were to deviate significantly from the exact solution. This is due to the fact that the linear B-spline wavelet has a vanishing first moment. Table I provides the average number of multiplications necessary to solve for the surface current of $S_{3}$ for both Haar and linear B-spline wavelets and for different values of threshold levels. It is found that the number of multiplications or, equivalently, the computation time, decreases significantly with the first order B-spline scaling function and its corresponding wavelets. For the Haar wavelet-based MoM, a slight improvement was observed (approximately 20\%), yet for the linear B-spline wavelet-based MoM, a factor of about 20 improvement was observed.

The question arises if the solution produced using a wavelet based MoM technique show the effect of backscattering enhancement. The backscattering enhancement is produces primarily due to multipath and surface-wave effects on a rough surface. A surface was chosen that has physical parameters where backscattering enhancement is known to occur and these parameters are $k s=10.636, k \ell=19.472$. Fig. 9 was produced using the aforementioned physical parameters as well as number of independent surfaces $N=400$, sample length $=30 \lambda$, resis-

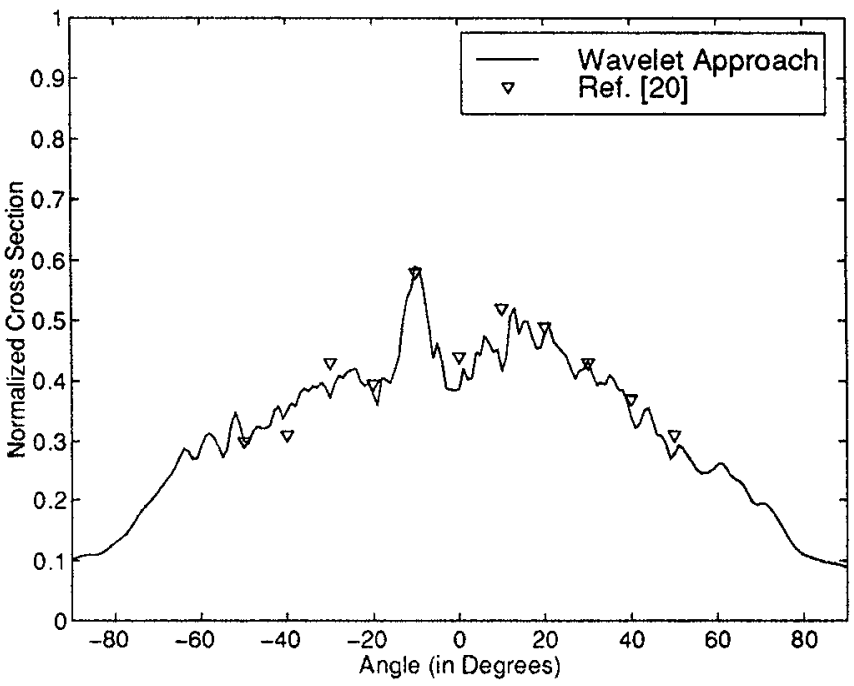

Fig. 9. Test for backscatter enhancement effect. The surfaces under test have $k s=10.636, k \ell=19.472, N=400$, length $=30 \lambda$, resistive tapered ends length $=1 \lambda, \Delta x=0.0625 \lambda$, and $\theta_{i}=10.0^{\circ}$. As can be seen, a significant backscatter enhancement is shown at $\theta_{s}=-10^{\circ}$.

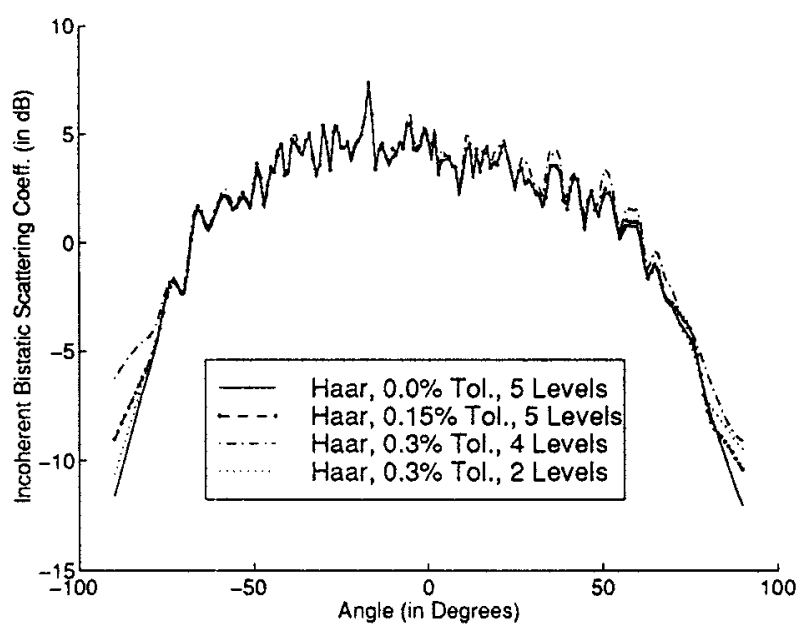

Fig. 10. Comparison of resolution levels used on scattering pattern for a random surface with $k s=2.0, k \ell=2.5, N=40$, length $=32 \lambda$, resistive tapered ends length $=1 \lambda, \Delta x=0.04 \lambda$, and $\theta_{i}=30.0^{\circ}$.

tive tapered ends length $=1 \lambda, \Delta x=0.0625 \lambda$, and $\theta_{i}=10.0^{\circ}$. Also, five levels of resolution were used, and the matrices had an average sparsity of $97.5 \%$. It can be seen that around $\theta_{s}=-10^{\circ}$ there is an enhanced backscattering effect. This figure is in linear scale to display the enhanced backscattering effect and agrees very well with [5, fig. 5]. It is also wise to point out that even though Figs. 7 and 10 show an increased bistatic scattering at about $20^{\circ}$, this is not an enhanced backscattering effect.

The effect of the number of resolution levels on the scattering pattern is next investigated using surface $S_{3}$. Since it has already been determined that both Haar and B-spline waveletbased MoM produce similar results, the effect of the number of resolution levels is demonstrated with Haar wavelets only. Monte Carlo simulations are run on random rough surfaces for five, four, and two levels of resolution for threshold levels of 0.1 
TABLE II

SPARSITY VERSUS THRESHOLD FOR A NUMBER OF DIFFERENT RESOLUTION LEVELS USED FOR A RANDOM SURFACE WITH $k s=2.0, k \ell=2.5$ $N=40$, LENGTH $=32 \lambda$, RESISTIVE TAPERED ENDS LENGTH $=1 \lambda$, $\Delta x=0.04 \lambda$ AND $\theta_{i}=30.0^{\circ}$

\begin{tabular}{c|c|c}
\hline & Threshold & Sparsity \\
\hline Haar & $0.0 \%$ & $0.0 \%$ \\
Haar-5 levels res. & $0.1 \%$ & $76.9 \%$ \\
Haar-5 levels res. & $0.15 \%$ & $82.2 \%$ \\
Haar-4 levels res. & $0.1 \%$ & $68.9 \%$ \\
Haar-4 levels res. & $0.3 \%$ & $85.5 \%$ \\
Haar-2 levels res. & $0.1 \%$ & $51.1 \%$ \\
Haar-2 levels res. & $0.3 \%$ & $74.4 \%$ \\
\hline
\end{tabular}

and $0.3 \%$, except for the Haar with five levels of resolution in which 0.1 and $0.15 \%$ threshold level is used. This is because $0.3 \%$ threshold level is too high for five levels of resolution and the conjugate gradient solver does not converge. The results based on the full matrix is also included at five levels of resolution for comparison. As is shown in Fig. 10 the scattering pattern starts deviating from the exact pattern for five levels of resolution and $0.1 \%$ threshold level imposed on the moment matrix. The average sparsity, obtained from 40 independent samples, for each case in Fig. 10 is summarized in Table II. The scattering pattern agrees quite well with the exact solution for almost all levels of resolutions shown with only slight deviation at near grazing observations. One notable exception is for four levels of resolution and an imposed tolerance level of $0.3 \%$, which deviates noticeably from the exact bistatic pattern at certain angles of observation. As shown in Table II, the matrix is less sparse for fewer levels of resolution at a single stated threshold level. Therefore, by increasing the number of resolution levels, while holding a constant threshold level, the sparsity of the matrix will increase, as is shown in Table II.

\section{CONCLUSION}

It has been found that using wavelet basis functions with MoM and Galerkin's method along with a fast solver routine such as conjugate gradient can drastically reduce both the memory requirements of a system and the time necessary to solve the MoM matrix. This leads to solutions for rough surface scattering that are quite accurate when compared to other basis functions, yet take significantly less memory and time to solve. Matrices can be made over $97 \%$ sparse yet still produce accurate bistatic scattering coefficients in scattering problems. Thus, it becomes possible to generate statistics for the scattering from surfaces of different roughness in a relatively short period of time.

The number of resolution levels were shown to play a significant role in determining the sparsity of the matrix and the accuracy of the solution. It was shown that the higher the number of resolutions, the more sparse the matrix could be made without compromising the bistatic scattering pattern. Also, the higher order the B-spline was made, the higher the sparsity achieved in the matrix and, thus, the faster the computation time to solve the matrix.

\section{REFERENCES}

[1] R. M. Axline and A. K. Fung, "Numerical computation of scattering from a perfectly conducting random surface," Trans. Antennas Propagat., vol. AP-26, pp. 482-488, May 1978.

[2] P. Beckmann and A. Spizzichino, The Scattering of Electromagnetic Waves from Rough Surfaces. Norwood, MA: Artech House, 1987.

[3] C. K. Chui, Ed., Wavelets-A Tutorial in Theory and Applications. New York: Academic, 1992.

[4] A. Ishimaru, Wave Propagation and Scattering in Random Media-Volume 2. New York: Academic, 1978.

[5] L. Li, C. H. Chan, L. Tsang, K. Pak, P. Phu, and S. H. Lou, "Monte Carlo simulations and backscattering enhancement of random metallic rough surfaces at optical frequencies," J. Electromagn. Waves Applicat., vol. 8, no. 3, pp. 277-293, 1994.

[6] M. Nieto-Vesperinas, "Depolarization of electromagnetic waves scattered from slightly rough random surfaces-A study by means of the extinction theorem," J. Opt. Soc. Amer., vol. 72, pp. 539-547, 1982.

[7] Y. Oh and K. Sarabandi, "An improved numerical simulation of electromagnetic scattering from perfectly conducting random surfaces," Proc. Inst. Elect. Eng. Microwave Antenna Propagation, to be published.

[8] Y. Oh, K. Sarabandi, and F. T. Ulaby, "Re-examination of the Kirchoff approximation for scattering from a rough surface," in URSI Radio Sci. Meet. Dig., Ann Arbor, MI, June 28-July 2 1993, p. 406.

[9] P. Phu, A. Ishimaru, and Y. Kuga, "Controlled millimeter-wave experiments and numerical simulations on the enhanced backscattering from one-dimensional very rough surfaces," Radio Sci., vol. 28, no. 4, pp. 533-548, July/Aug. 1993.

[10] S. O. Rice, "Reflection of electromagnetic waves by slightly rough surfaces," Commun. Pure Appl. Math., vol. 4, pp. 357-378, 1951.

[11] O. Rioul and P. Duhamel, "Fast algorithms for discrete and continuous wavelet transforms," IEEE Trans. Inform. Theory, vol. 38, pp. 569-586, Mar. 1992.

[12] E. Rodriguez, Y. Kim, and S. L. Durden, "A numerical assessment of rough surface scattering theories-Horizontal polarization," Radio Sci., vol. 27, pp. 497-513, July/Aug. 1992.

[13] K. F. Sabet and P. B. Katehi, "Analysis of integrated millimeter-wave and submillimeter-wave waveguides using orthonormal wavelet expansions," IEEE Trans. Microwave Theory Tech., vol. 42, Dec. 1994.

[14] K. F. Sabet, "Novel efficient integral-based techniques for characterization of planar microwave structures," Ph.D. dissertation, Univ. Michigan, Ann Arbor, MI, Apr. 1995.

[15] — - "Novel efficient integral-based techniques for characterization of planar microwave structures," Ph.D. dissertation, Univ. Michigan, Ann Arbor, MI, 1995.

[16] K. F. Sabet and P. B. Katehi, "A study of dielectric resonators based on two-dimensional fast wavelet algorithm," IEEE Microwave Guided Wave Lett., vol. 6, pp. 19-21, Jan. 1996.

[17] K. F. Sabet, J.-C. Cheng, and L. P. B. Katehi, "Efficient wavelet-based modeling of printed circuit antenna arrays," IEE Proc. Microwave Antennas Propagat., vol. 146, no. 4, Aug. 1999, to be published.

[18] K. Sarabandi and Y. Oh, "Effect of antenna footprint on the statistics of radar backscattering," in IEEE Trans. Geosci. Remote Sensing Symp., Firenze, Italy, July 1995.

[19] K. Sarabandi, Y. Oh, and F. T. Ulaby, "A numerical simulation of scattering from one-dimensional inhomogeneous dielectric random surfaces," IEEE Trans. Geosci. Remote Sensing, vol. 34, pp. 425-432, Mar. 1996.

[20] T. B. A. Senior, "Scattering by resistive strips," Radio Sci., vol. 14, pp. 911-924, 1979.

[21] B. Z. Steinberg and Y. Leviatan, "On the use of wavelet expansions in the method of moments," IEEE Trans. Antennas Propagat., vol. 41, pp. 610-619, May 1993.

[22] E. I. Thorsos, "The validity of the Kirchhoff approximation for rough surface scattering using a Gaussian roughness spectrum," J. Acoust. Soc. Amer., vol. 83, pp. 78-92, Jan. 1988.

[23] E. I. Thorsos and D. R. Jackson, "The validity of the perturbation approximation for rough surface scattering using a Gaussian roughness spectrum," J. Acoust. Soc. Amer., vol. 86, pp. 261-276, July 1989.

[24] L. Tsang, J. A. Kong, and R. T. Shin, Theory of Microwave Remote Sensing. New York: Wiley, 1985. 


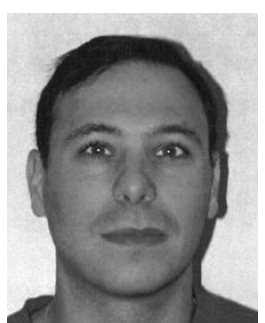

Daniel Zahn received the S.B. and M. Eng. degrees from the Massachusetts Institute of Technology, Cambridge, MA, in 1995. He is currently working toward the Ph.D. degree at the University of Michigan, Ann Arbor.

His interests include scattering from random rough surfaces, experimental bistatic scattering measurements, polarimetric radar systems, calibration and measurement techniques, and microwave circuits. In addition, he has done extensive work in numerical methods for predicting scattering from

random rough surfaces

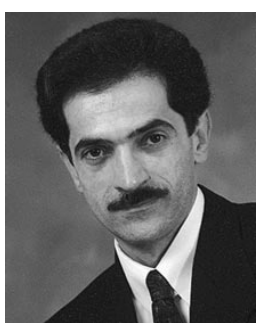

Kamal Sarabandi (S'87-M'90-SM'92) received the B.S. degree in electrical engineering from Sharif University of Technology, Tehran, Iran, in 1980, the M.S.E. degree in electrical engineering from the University of Michigan, Ann Arbor, in 1986, and both the M.S. (mathematics) and Ph.D. (electrical engineering) degrees from the University of Michigan, Ann Arbor, in 1989.

From 1980 to 1984 , he worked as a Microwave Engineer in the Telecommunication Research Center. $\mathrm{He}$ is currently an Associate Professor in the Department of Electrical Engineering and Computer Science, University of Michigan. $\mathrm{He}$ has 18 years of experience with microwave sensors and radar systems. In the past eight years he has served as the Principal Investigator and Co-Investigator on many projects sponsored by NASA, JPL, ARO, ONR, ARL, and GM, all related to microwave and millimeter-wave radar remote sensing. He has published many book chapters and more than 80 papers in refereed journals on electromagnetic scattering, random media modeling, microwave measurement techniques, radar calibration, application of neural networks in inverse scattering problems, and microwave sensors. He has also had more than 140 papers and invited presentations in national and international conferences and symposia on similar subjects.

Dr. Sarabandi is listed in American Men and Women of Science and Who's Who in Electromagnetics. He is a member of the IEEE Geoscience and Remote Sensing ADCOM since January 1998 and served as the Chairman of Geoscience and Remote Sensing Society Southeastern Michigan chapter from 1992 to 1998 $\mathrm{He}$ is also a Member of Commission F of URSI and of the Electromagnetic Academy. He was a recipient of a 1996 Teaching Excellence Award, the 1997 Henry Russel Award from the Regent of the University of Michigan, and the 1999 GAAC Distinguished Lecturer Award from the German Federal Ministry for Education, Science, and Technology.

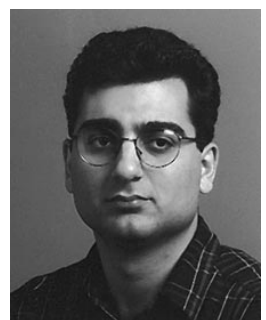

Kazem F. Sabet (S'86-M'95) received the Ph.D. degree in electrical engineering from the University of Michigan in 1995.

His doctoral research concerned the efficient modeling of planar microwave and millimeter wave circuits and antennas, including novel wavelet techniques for large-scale electromagnetic structures. He has since presented several workshops on the applications of wavelet theory to numerical modeling of electromagnetic problems. $\mathrm{He}$ is currently the President of EMAG Technologies, a Michigan based research and development company that provides innovative hardware/software solutions for microwave and wireless technologies. He has served as the Principal Investigator of numerous government and industry research and development projects on antenna technology. Many of these projects have successfully brought advanced research concepts into commercial use. His research interests include novel microwave circuits and antennas, computational electromagnetics and high frequency computer-aided design (CAD) development.

Dr. Sabet is a member of Tau Beta Pi. His doctoral research won him three IEEE Student Paper Awards.

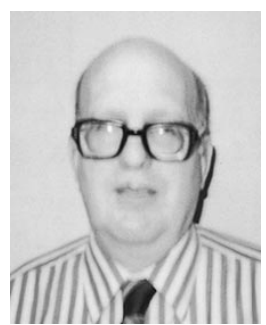

James F. Harvey (M'91-SM'99) received the B.S. degree in engineering from the United States Military Academy, West Point, NY, in 1964, the M.A. degree in physics from Dartmouth College, Hanover, $\mathrm{NH}$, in 1972, and the Ph.D. degree in applied science from the University of California at Davis, in 1990, with research performed at Lawrence Livermore National Laboratory.

He served in a variety of electrical engineering and research assignments as a member of the United States military before retiring. He is currently a Civilian Research Program Manager at the U.S. Army Research Office, Research Triangle Park, NC, with primary responsibility for the fields of electromagnetics, antennas and antenna structures, innovative microwave and millimeter-wave circuit integration, low-power/minimum-power system design, and landmine detection. His programs include a focus on small multifrequency multifunctional antennas for army vehicles, radio propagation over complex terrain affecting data communications, new millimeter-wave circuit integration techniques such as spatial power combining, micromachining, and advanced electromagnetic calculational techniques. His personal research interests are in the fields of quasi-optics, radio wave propagation, and multiresolution analysis of electromagnetic structures.

Dr. Harvey is an active member in the IEEE Microwave Theory and Techniques Society, the IEEE Antennas and Propagation Society, URSI Commission D, and SPIE. He is an organizer of the annual conference on the Detection and Remediation Technologies for Mines and Minelike Targets in the SPIE Aerosense Meeting and he is an Editor of those Conference Proceedings. He received the Army R\&D Award in 1992 